\title{
Ensemble approach to coherence between two scalar harmonic light vibrations and the phase difference
}

\author{
Alfredo Luis* \\ Departamento de Óptica, Facultad de Ciencias Físicas, Universidad Complutense, 28040 Madrid, Spain
}

(Received 15 March 2009; published 28 May 2009)

\begin{abstract}
We develop an approach to coherence between two scalar harmonic light vibrations derived from the ensemble interpretation of statistical optics. Coherence is presented as a statistical variable itself that turns out to be the phase difference between the two vibrations. This provides a natural and simple extension of second-order coherence to cover more complicated situations. This includes in a single formalism both classic and quantum light states, allowing the most accurate interferometric measurements, even if they are incoherent according to the standard second-order approach.
\end{abstract}

DOI: 10.1103/PhysRevA.79.053855

PACS number(s): 42.25.Kb, 42.25.Hz, 42.50.St

\section{INTRODUCTION}

Coherence is a key concept in optics derived from the statistical nature of real light beams [1-4]. For definiteness we consider two scalar harmonic light vibrations that may be representing the light amplitudes at the two pinholes of a Young's interferometer as well as the amplitudes of two field modes at the input ports of the Mach-Zehnder interferometer, for example.

The motive for this work is that the standard second-order degree of coherence $\mu$ is insufficient to address some relevant situations beyond thermal Gaussian light. This is the case in quantum metrology, where light states providing the maximum interferometric resolution allowed by the quantum theory have $\mu=0$ [5-9]. This is caused by symmetry properties of the field states instead of by a large amount of fluctuations. A key point is that such powerful interferometric schemes go beyond second-order optics by employing nonlinear detection schemes.

In this work we develop an alternative approach by identifying coherence with the phase difference between the two vibrations. This choice is aimed to include in a single formalism both classic second-order optics and recent quantum interferometric schemes. The two main reasons supporting the soundness of this objective are the following:

(i) This may be regarded as an approximation ascribing partial coherence to the result of phase fluctuations. This is customary in classical optics since in most situations partial visibility of interference fringes can be explained just in terms of phase fluctuations rather than amplitude fluctuations.

(ii) This is an exact approach from an ensemble perspective defining coherence for each sample realization and then statistically averaging over sample repetitions.

The identification of coherence as a statistical variable allows derivation of a probability distribution that contains information about coherence to all orders. Thus, a key feature of this approach is that it provides a simple and natural extension of coherence beyond second-order optics without involving an infinite hierarchy of correlation functions. We

\footnotetext{
*alluis@ fis.ucm.es; URL: http://www.ucm.es/info/gioq
}

show below that the alternative and standard approaches coincide when applied to common thermal light, but lead to opposite results when applied to more sophisticated light states.

In Sec. II we show that the ensemble approach leads to identification of coherence with phase difference. In Sec. III we consider the assessment of the amount of coherence within this formalism. In Sec. IV we apply this approach to some relevant examples, such as thermal light and quantum states reaching optimum interferometric resolution.

\section{ENSEMBLE COHERENCE}

We consider two harmonic scalar vibrations (two field modes) that are statistically fully described by two scalar random complex amplitudes, $E_{1}$ and $E_{2}$. The second-order complex degree of coherence between two scalar electromagnetic vibrations $E_{1,2}$ is introduced as a function of averages of complex-amplitude products $\left\langle\left|E_{1}\right|^{2}\right\rangle,\left\langle\left|E_{2}\right|^{2}\right\rangle$, and $\left\langle E_{1}^{*} E_{2}\right\rangle[1-4]$,

$$
\mu=\frac{\left\langle E_{1}^{*} E_{2}\right\rangle}{\sqrt{\left\langle\left|E_{1}\right|^{2}\right\rangle\left\langle\left|E_{2}\right|^{2}\right\rangle}} .
$$

\section{A. Coherence and phase difference}

In this work coherence is presented as a statistical variable itself, rather than as a function of averages of other variables. From the ensemble-average picture this can be done by defining coherence for each sample realization and then deriving the corresponding probability distribution describing its random variation when the ensemble member is varied.

Every field realization provides a pair of definite complex amplitudes $E_{1}(j)$ and $E_{2}(j)$, where $j$ indexes the sample. The single-realization counterpart of Eq. (2.1) is

$$
\mu_{e}(j)=\frac{E_{1}^{*}(j) E_{2}(j)}{\sqrt{\left|E_{1}(j)\right|^{2}\left|E_{2}(j)\right|^{2}}}=\exp \left(i \phi_{j}\right),
$$

where $\phi_{j}=\arg \left[E_{2}(j)\right]-\arg \left[E_{1}(j)\right]$ is the phase difference between $E_{1}(j)$ and $E_{2}(j)$. This result agrees with common physical intuition since in most situations partial visibility of 
interference fringes can be explained just in terms of phase fluctuations rather than amplitude fluctuations.

Although all the samples have maximum coherence $\left|\mu_{e}(j)\right|=1$, the ensemble will not in general, because the phase $\phi_{j}$ will change randomly from realization to realization so that $\left|\left\langle\mu_{e}\right\rangle\right| \leq 1$. A similar ensemble approach arises in the polarization context where partial polarization is naturally regarded as the random succession of different fully polarized states [10-13].

Incidentally, $\mu$ can be expressed also as an average of phase difference in the context of the Wigner-function formulation of paraxial optics, as shown in Ref. [14]. Further relations between coherence and phase in the quantum and classical domains can be found in Ref. [15].

\section{B. Probability distribution}

When the sample realization is repeated, the complex amplitudes $E_{1}(j)$ and $E_{2}(j)$ change so that the ensemble average of any quantity $F$ is

$$
\langle F\rangle=\lim _{N \rightarrow \infty} \frac{1}{N} \sum_{j=1}^{N} F\left[E_{1}(j), E_{2}(j)\right],
$$

where $N$ is the number of ensemble members. The variability of $E_{1}(j)$ and $E_{2}(j)$ can be suitably represented by a probability distribution $P\left(E_{1}, E_{2}\right)$ expressing the probability of appearance of each pair of complex amplitudes $\left(E_{1}, E_{2}\right)$ throughout the ensemble. This probability distribution allows computation of the same ensemble average (2.3) as

$$
\langle F\rangle=\int d^{2} E_{1} d^{2} E_{2} F\left(E_{1}, E_{2}\right) P\left(E_{1}, E_{2}\right) .
$$

Moreover, complete information about $F$ is provided by the marginal probability distribution $P(f)$, which can be derived from $P\left(E_{1}, E_{2}\right)$ as

$$
P(f)=\int d^{2} E_{1} d^{2} E_{2} \delta\left[f-F\left(E_{1}, E_{2}\right)\right] P\left(E_{1}, E_{2}\right),
$$

where $\delta$ is the Dirac delta function. This allows computation of the same ensemble averages (2.3) and (2.4) as

$$
\langle F\rangle=\int d f f P(f) .
$$

Next we apply this general approach to the phase difference $f=\phi, F=\arg E_{2}-\arg E_{1}$. To derive $P(\phi)$ from $P\left(E_{1}, E_{2}\right)$ it will be useful to consider different parametrizations for the complex amplitudes $E_{1}$ and $E_{2}$ in order to simplify as far as possible the integrals in Eq. (2.5). The choice of the proper parametrization depends on the particular $P\left(E_{1}, E_{2}\right)$ considered. For definiteness we will consider two possibilities to be used in the examples in Sec. IV. In any case the idea is to include $\phi$ as part of the coordinates describing $E_{1}$ and $E_{2}$. Then to obtain $P(\phi)$ one must integrate $P\left(E_{1}, E_{2}\right)$ over all the variables except $\phi$ itself.

Usually the complex amplitudes $E_{1}$ and $E_{2}$ are described by real Cartesian coordinates representing their real and imaginary parts,

$$
x_{1,2}=\frac{1}{2}\left(E_{1,2}+E_{1,2}^{*}\right), \quad y_{1,2}=\frac{i}{2}\left(E_{1,2}^{*}-E_{1,2}\right),
$$

or

$$
E_{1,2}=x_{1,2}+i y_{1,2}
$$

with

$$
d^{2} E_{1} d^{2} E_{2}=d x_{1} d y_{1} d x_{2} d y_{2} .
$$

Alternatively, we will find useful to use two different expressions for $E_{1}$ and $E_{2}$. The first one is

$$
E_{1}=r \cos \frac{\theta}{2} \exp (i \xi), \quad E_{2}=r \sin \frac{\theta}{2} \exp (i \phi) \exp (i \xi)
$$

with $r \geq 0, \pi \geq \theta \geq 0, \pi \geq \phi, \xi \geq-\pi$, and

$$
d^{2} E_{1} d^{2} E_{2}=\frac{1}{4} r^{3} \sin \theta d r d \theta d \phi d \xi .
$$

More explicitly, the field variables $r, \theta, \xi$, and $\phi$ are defined as

$$
\begin{gathered}
r=\sqrt{\left|E_{1}\right|^{2}+\left|E_{2}\right|^{2}}, \quad \theta=2 \arctan \left|\frac{E_{2}}{E_{1}}\right|, \\
\phi=\arg E_{2}-\arg E_{1}, \quad \xi=\arg E_{1} .
\end{gathered}
$$

The meaning of $r^{2}$ is total intensity, $\xi$ is the phase of $E_{1}$, and $\phi$ is the phase difference between $E_{2}$ and $E_{1}$. The angle $\theta$ expresses the distribution of the total intensity $r^{2}$ between the two fields $E_{1}$ and $E_{2}$, since $\left|E_{1}\right|^{2}=r^{2} \cos ^{2}(\theta / 2)$ and $\left|E_{2}\right|^{2}$ $=r^{2} \sin ^{2}(\theta / 2)$. (The factor of $1 / 2$ is introduced for convenience to simplify formulas and has no any further meaning.) With this parametrization (2.5) leads to the following formula for $P(\phi)$ :

$$
P(\phi)=\frac{1}{4} \int_{-\pi}^{\pi} d \xi \int_{0}^{\infty} d r r^{3} \int_{0}^{\pi} d \theta \sin \theta P\left(E_{1}, E_{2}\right),
$$

where $E_{1}, E_{2}$ in $P\left(E_{1}, E_{2}\right)$ should be expressed as in Eq. (2.10).

The second particular parametrization we will find useful is

$$
E_{1}=r_{1} \exp (i \xi), \quad E_{2}=r_{2} \exp (i \phi) \exp (i \xi),
$$

with $r_{1,2} \geq 0, \pi \geq \phi, \xi \geq-\pi$, and

$$
d^{2} E_{1} d^{2} E_{2}=r_{1} r_{2} d r_{1} d r_{2} d \phi d \xi .
$$

More explicitly, the field variables $r_{1}, r_{2}, \xi$, and $\phi$ are defined as

$$
\begin{gathered}
r_{1}=\left|E_{1}\right|, \quad r_{2}=\left|E_{2}\right|, \\
\phi=\arg E_{2}-\arg E_{1}, \quad \xi=\arg E_{1} .
\end{gathered}
$$

In this case $r_{1}^{2}$ and $r_{2}^{2}$ represent the individual intensities. The formula used to obtain $P(\phi)$ in this case is 


$$
P(\phi)=\int_{-\pi}^{\pi} d \xi \int_{0}^{\infty} d r_{1} r_{1} \int_{0}^{\infty} d r_{2} r_{2} P\left(E_{1}, E_{2}\right)
$$

Note that to describe the two scalar complex amplitudes $E_{1}$ and $E_{2}$, we need four real quantities. They can be the four Cartesian variables in Eq. (2.7), the three angles and a radius in Eq. (2.10), or the two angles and two radii in Eq. (2.14).

\section{DEGREE OF COHERENCE}

Within this approach fully incoherent light is represented by a uniform phase-difference distribution $P_{\text {incoherent }}(\phi)$ $=1 / 2 \pi$. Thus, the amount of coherence can be assessed in terms of the distance $D$ between $P(\phi)$ and the uniform distribution $1 / 2 \pi$ as

$$
D=2 \pi \int_{-\pi}^{\pi} d \phi\left[P(\phi)-\frac{1}{2 \pi}\right]^{2} .
$$

Since $D$ is unbounded we can construct a normalized degree of coherence $\gamma$, with $0 \leq \gamma \leq 1$, as

$$
\gamma=\sqrt{\frac{D}{D+1}} .
$$

This formulation is consistent with similar definitions of other optical properties [13,15-17].

Note that $\gamma$ presents the same invariance properties as $\mu$. This is because $\gamma$ and $\mu$ are invariant under nonrandom invertible complex scale changes $E_{1,2} \rightarrow \eta_{1,2} E_{1,2}$ for complex $\eta_{1,2} \neq 0$. In our case these transformations produce just a phase shift $P(\phi) \rightarrow P(\phi+$ constant $)$ that does not affect the value of $\gamma$.

It is shown below that for Gaussian fields $\gamma \simeq|\mu|$. However, in the general case $\gamma \neq|\mu|$ since they correspond to different statistical evaluations. More specifically, $\mu$ is a second-order quantity on the complex field amplitudes $E_{1,2}$, while $\gamma$ depends exclusively on the statistics of the phase difference $\phi$. This is a nonlinear function of $E_{1,2}$ so that $\gamma$ goes beyond second-order statistics by depending on all the moments of $P\left(E_{1}, E_{2}\right)$.

Within this approach coherence is naturally determined by the amount of fluctuations of the phase difference. Since this is an angular variable the assessment of fluctuations is not as simple as for Cartesian variables $[18,19]$. In our case we have

$$
D=\frac{1}{\Delta \phi}-1, \quad \gamma=\sqrt{1-\Delta \phi},
$$

where

$$
\Delta \phi=\frac{1}{2 \pi \int_{-\pi}^{\pi} d \phi P^{2}(\phi)}
$$

is the Süssmann measure [19]. This is also the particular case $\alpha=2$ of the Rényi entropies [20,21]

$$
\mathcal{S}_{\alpha}[P]=\frac{1}{1-\alpha} \ln \left\{\int_{-\pi}^{\pi}[P(\phi)]^{\alpha} d \phi\right\} .
$$

Equivalent expressions have been used in different contexts as measures of localization, uncertainty, and information $[19,21]$.

\section{EXAMPLES}

In this section we present simple and meaningful examples showing that the ensemble approach provides meaningful results in very different contexts.

\section{A. Gaussian}

A very common particular case is thermal light described by a zero-mean Gaussian distribution for the complex amplitudes,

$$
P_{G}\left(E_{1}, E_{2}\right)=\frac{1}{\pi^{2} \operatorname{det} \boldsymbol{\Phi}} \exp \left(-\boldsymbol{E}^{\dagger} \boldsymbol{\Phi}^{-1} \boldsymbol{E}\right),
$$

where $\boldsymbol{E}^{t}=\left(E_{1}, E_{2}\right)$,

$$
\boldsymbol{\Phi}=\left(\begin{array}{cc}
\left\langle\left|E_{1}\right|^{2}\right\rangle & \left\langle E_{1} E_{2}^{*}\right\rangle \\
\left\langle E_{1}^{*} E_{2}\right\rangle & \left\langle\left|E_{2}\right|^{2}\right\rangle
\end{array}\right)=\left(\begin{array}{cc}
I_{1} & \sqrt{I_{1} I_{2}} \mu^{*} \\
\sqrt{I_{1} I_{2}} \mu & I_{2}
\end{array}\right),
$$

with $I_{j}=\left\langle\left|E_{j}\right|^{2}\right\rangle$ and $\operatorname{det} \boldsymbol{\Phi}=I_{1} I_{2}\left(1-|\mu|^{2}\right)$, and we have assumed det $\boldsymbol{\Phi} \neq 0$ (below we consider the case $\operatorname{det} \boldsymbol{\Phi}=0$ ). After two consecutive changes of variables, which are Eq. (2.14) and

$$
x=\sqrt{\frac{I_{2}}{\operatorname{det} \boldsymbol{\Phi}}} r_{1}, \quad y=\sqrt{\frac{I_{1}}{\operatorname{det} \boldsymbol{\Phi}}} r_{2},
$$

the integrations in Eq. (2.17) can be expressed as

$$
\begin{aligned}
P_{G}(\phi)= & 2 \frac{1-|\mu|^{2}}{\pi} \int_{0}^{\infty} d x \int_{0}^{\infty} d y x y \\
& \times \exp \left(-x^{2}-y^{2}+2 x y|\mu| \cos \phi\right),
\end{aligned}
$$

where we have assumed without loss of generality that $\mu$ is real. After expanding $\exp (2 x y|\mu| \cos \phi)$ in a power series, we get finally

$$
P_{G}(\phi)=\sum_{n=0}^{\infty} c_{n} \cos ^{n} \phi, \quad c_{n}=\frac{1-|\mu|^{2}}{2 \pi} \frac{2^{n} \Gamma^{2}\left(\frac{n}{2}+1\right)}{n !}|\mu|^{n},
$$

where $\Gamma$ is the gamma function. In Fig. 1 we have represented probability distribution (4.5) for $|\mu|=0.9$.

The degree of coherence $\gamma$ in Eq. (3.2) is given by

$$
\int_{-\pi}^{\pi} d \phi P_{G}^{2}(\phi)=2 \sqrt{\pi} \sum_{k, \ell=0}^{\infty} c_{k} c_{\ell} \frac{\Gamma\left(\frac{k+\ell+1}{2}\right)}{\left(\frac{k+\ell}{2}\right) !} \cos ^{2}\left[\frac{(k+\ell) \pi}{2}\right],
$$

where the factor $\cos ^{2}[(k+\ell) \pi / 2]$ is introduced to select only the contribution of the terms with even $k+\ell$. In Fig. 2 we 


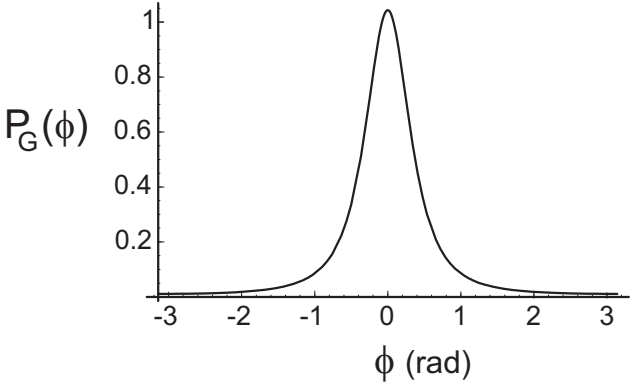

FIG. 1. Probability distribution (4.5) for a Gaussian field state with $|\mu|=0.9$.

have represented $\gamma$ as a function of $|\mu|$ where it can appreciated that for Gaussian fields $\gamma \simeq|\mu|$.

For completeness we finally consider the case $\operatorname{det} \boldsymbol{\Phi}=0$, which corresponds to the amplitude distribution

$$
P\left(E_{1}, E_{2}\right)=\frac{\eta}{\pi} \exp \left(-\eta\left|A_{1}\right|^{2}\right) \delta\left(A_{2}\right),
$$

where $A_{1,2}$ are complex linear combinations of the original amplitudes $E_{1,2}$, such as $A_{2}=\alpha E_{1}+\beta E_{2}$.

When $\operatorname{det} \boldsymbol{\Phi}=I_{1} I_{2}\left(1-|\mu|^{2}\right)=0$ we have either (i) $|\mu|=1$ or (ii) $I_{1} I_{2}=0$. For case (i) we have $\alpha \beta \neq 0$ and the delta function $\delta\left(\alpha E_{1}+\beta E_{2}\right)$ in Eq. (4.7) means that the phase difference is fixed and not fluctuating, $P_{G}(\phi)=\delta\left(\phi-\phi_{0}\right)$, where $\phi_{0}=\arg \alpha-\arg \beta+\pi$. Thus $\gamma=1$, in agreement with $|\mu|=1$. In case (ii) $I_{1}=0$ or $I_{2}=0$; both $\mu$ and $\mu_{e}$ are undefined since coherence requires the existence of two nonvanishing electric fields to be defined.

\section{B. Twin photon states}

As a more sophisticated example let us consider a field state with quantum metrological applications. This is a twin photon-number state $|n\rangle_{1}|n\rangle_{2}$ with $n$ photons in mode $E_{1}$ and $n$ photons in mode $E_{2}[5,6]$. More specifically we will focus on its coherence properties after passing through a $50 \%$ lossless beam splitter, which can be represented as the input beam splitter of the Mach-Zehnder interferometer as illustrated in Fig. 3.

This example is of relevance since for this state $\mu=0$ although it reaches the maximum interferometric precision allowed by the quantum theory (the Heisenberg limit). This paradox is solved by the ensemble approach to coherence developed in this work.

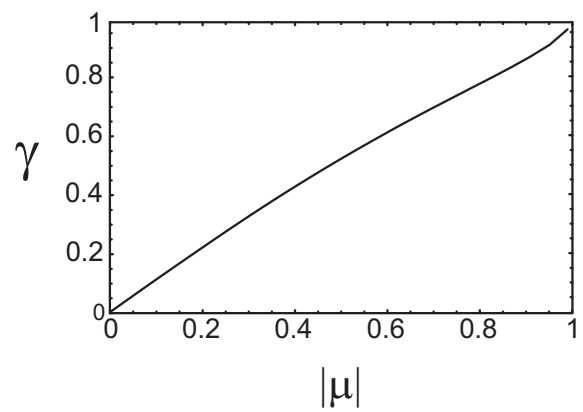

FIG. 2. $\gamma$ as a function of $|\mu|$ for a Gaussian field state.

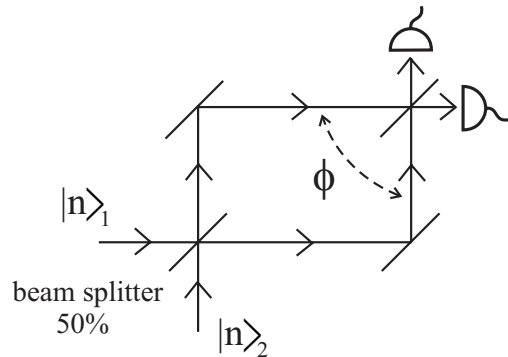

FIG. 3. Scheme of a Mach-Zehnder interferometer illuminated by the twin photon-number state $|n\rangle_{1}|n\rangle_{2}$.

As a suitable field distribution $P\left(E_{1}, E_{2}\right)$ we consider the $Q$ function, defined by projection of the field state on coherent states $[2,22]$. This means that ensemble coherence becomes the classical version of the quantum phase-difference approach based on the $Q$ function [23]. In such a case the amplitude distribution for the state $|n\rangle_{1}|n\rangle_{2}$ before the beam splitter is (assuming $E_{1,2}$ to be dimensionless by a suitable scaling)

$$
P_{n}\left(E_{1}, E_{2}\right)=\left(\frac{1}{\pi n !}\right)^{2}\left|E_{1} E_{2}\right|^{2 n} \exp \left(-\left|E_{1}\right|^{2}-\left|E_{2}\right|^{2}\right) .
$$

After a 50\% lossless beam splitter performing the transformation

$$
E_{1} \rightarrow \frac{1}{\sqrt{2}}\left(E_{1}+E_{2}\right), \quad E_{2} \rightarrow \frac{1}{\sqrt{2}}\left(E_{1}-E_{2}\right),
$$

the field distribution becomes

$$
P_{n}\left(E_{1}, E_{2}\right)=\left(\frac{1}{\pi n !}\right)^{2} \frac{\left|E_{1}^{2}-E_{2}^{2}\right|^{2 n}}{2^{2 n}} \exp \left(-\left|E_{1}\right|^{2}-\left|E_{2}\right|^{2}\right) .
$$

After change of variables (2.10) we get

$$
P_{n}\left(E_{1}, E_{2}\right)=\left(\frac{1}{\pi n !}\right)^{2}\left(\frac{r^{2}}{2}\right)^{2 n}\left(1-\sin ^{2} \theta \cos ^{2} \phi\right)^{n} \exp \left(-r^{2}\right) .
$$

By expanding $1-\sin ^{2} \theta \cos ^{2} \phi$ in power series and performing the $r$ and $\theta$ integrations, we get the phase-difference distribution

$$
P_{n}(\phi)=\sum_{k=0}^{n} c_{k} \cos ^{2 k} \phi, \quad c_{k}=\frac{(2 n+1) !}{\sqrt{\pi} 4^{n+1} n !} \frac{(-1)^{k}}{(n-k) ! \Gamma(k+3 / 2)} .
$$

In Fig. 4 we have represented $P_{n}(\phi)$ for $n=20$. We can appreciate a double-peak structure that was already noticed in the quantum case using a different phase approach [6].

For this example $\mu=0$ since $P_{n}\left(-E_{1}, E_{2}\right)=P_{n}\left(E_{1}, E_{2}\right)$ so that $\left\langle E_{1} E_{2}^{*}\right\rangle=0$. Note that this holds by symmetry properties of $P_{n}\left(E_{1}, E_{2}\right)$ instead of being the result of a large amount of fluctuations. On the other hand $\gamma \neq 0$ since 


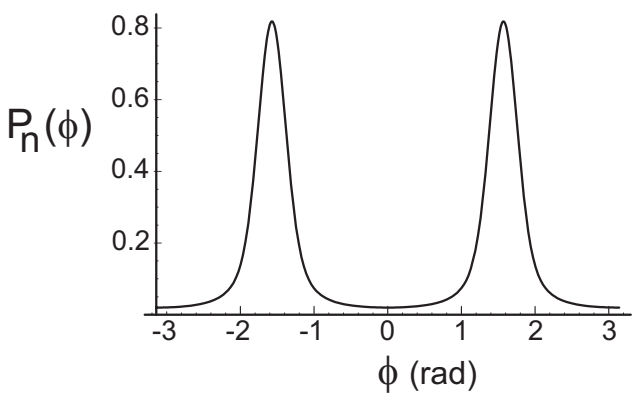

FIG. 4. Probability distribution (4.12) for a twin photon-number state $|n\rangle_{1}|n\rangle_{2}$ with $n=20$.

$$
\int_{2 \pi} d \phi P_{n}^{2}(\phi)=2 \sqrt{\pi} \sum_{k, \ell=0}^{n} c_{k} c_{\ell} \frac{\Gamma(k+\ell+1 / 2)}{(k+\ell) !} .
$$

In Fig. 5 we have represented $\gamma$ as a function of $n$. The degree of coherence $\gamma$ approaches its maximum value $\gamma$ $\rightarrow 1$ as $n$ increases, in agreement with the interferometric usefulness of these states.

\section{CONCLUSIONS}

We have developed an alternative approach to coherence by regarding it as a statistical variable, in agreement with an ensemble-average interpretation of statistical optics. Such ensemble coherence turns out to be equivalent to phase difference.

This approach is of interest since it includes in a single formalism both classic and quantum light states, allowing precise interferometric measurements. The ensemble and

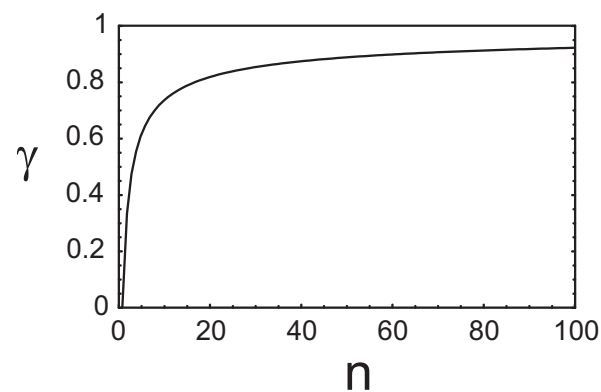

FIG. 5. $\gamma$ as a function of $n$ for the twin photon-number state $|n\rangle_{1}|n\rangle_{2}$

standard approaches coincide when applied to common Gaussian light, but lead to opposite results when applied to quantum light, providing the maximum resolution in interferometric measurements. Such states have vanishing standard degree of coherence, while they are highly coherent in the alternative formalism, in agreement with their interferometric usefulness.

This consistent inclusion of very different situations arises because this formalism is naturally an all-order approach. The application of the ensemble strategy to coherence regarded as a statistical variable leads to a probability distribution for coherence that contains information to all orders.

\section{ACKNOWLEDGMENTS}

This work was supported by Project No. FIS2008-01267 of the Spanish Dirección General de Investigación del Ministerio de Ciencia e Innovación.
[1] M. Born and E. Wolf, Principles of Optics, 7th expanded ed. (Cambridge University Press, Cambridge, England, 1999).

[2] L. Mandel and E. Wolf, Optical Coherence and Quantum Optics (Cambridge University Press, Cambridge, England, 1995).

[3] Ch. Brosseau, Fundamentals of Polarized Light: A Statistical Optics Approach (Wiley, New York, 1998).

[4] J. W. Goodman, Statistical Optics (Wiley, New York, 1985).

[5] M. J. Holland and K. Burnett, Phys. Rev. Lett. 71, 1355 (1993); P. Bouyer and M. A. Kasevich, Phys. Rev. A 56, R1083 (1997); T. Kim, O. Pfister, M. J. Holland, J. Noh, and J. L. Hall, ibid. 57, 4004 (1998); 58, 2617 (1998); T. Kim, J. Shin, Y. Ha, H. Kim, G. Park, T. G. Noh, and C. K. Hong, Opt. Commun. 156, 37 (1998); T. Kim, Y. Ha, J. Shin, H. Kim, G. Park, K. Kim, T.-G. Noh, and Ch. K. Hong, Phys. Rev. A 60, 708 (1999); V. Meyer, M. A. Rowe, D. Kielpinski, C. A. Sackett, W. M. Itano, C. Monroe, and D. J. Wineland, Phys. Rev. Lett. 86, 5870 (2001); J. A. Dunningham, K. Burnett, and S. M. Barnett, ibid. 89, 150401 (2002); H. Uys and P. Meystre, Phys. Rev. A 76, 013804 (2007); F. W. Sun, B. H. Liu, Y. X. Gong, Y. F. Huang, Z. Y. Ou, and G. C. Guo, EPL 82, 24001 (2008).

[6] M. Hillery, M. Zou, and V. Bužek, Quantum Semiclassic. Opt. 8, 1041 (1996).
[7] J. J. Bollinger, W. M. Itano, D. J. Wineland, and D. J. Heinzen, Phys. Rev. A 54, R4649 (1996).

[8] S. F. Huelga, C. Macchiavello, T. Pellizzari, A. K. Ekert, M. B. Plenio, and J. I. Cirac, Phys. Rev. Lett. 79, 3865 (1997).

[9] A. Rivas and A. Luis, Phys. Rev. A 77, 022105 (2008).

[10] R. Barakat, Opt. Acta 32, 295 (1985).

[11] A. T. Walden and T. Madkour, Proc. R. Soc. London, Ser. A 463, 3375 (2007).

[12] H. Hurwitz, Jr., J. Opt. Soc. Am. 35, 525 (1945).

[13] A. Luis, Phys. Rev. A 66, 013806 (2002); Opt. Commun. 216, 165 (2003); Phys. Rev. A 69, 023803 (2004); 71, 053801 (2005); 71, 063815 (2005); 73, 063806 (2006); Opt. Commun. 273, 173 (2007); Phys. Rev. A 75, 053806 (2007).

[14] A. Luis, Eur. J. Phys. 28, 231 (2007); Phys. Rev. A 76, 043827 (2007).

[15] A. Luis, Phys. Rev. A 78, 025802 (2008).

[16] A. Luis, J. Opt. Soc. Am. A 24, 1063 (2007); in Advances in Information Optics and Photonics, ICO Vol. VI, edited by A. T. Friberg and R. Dändliker (SPIE, Bellingham, WA, 2008), pp. 171-188.

[17] A. Luis, J. Phys. A 35, 8805 (2002); Phys. Lett. A 314, 197 (2003); Opt. Commun. 253, 10 (2005); 266, 426 (2006); J. Opt. Soc. Am. A 24, 2070 (2007). 
[18] J. M. Lévy-Leblond, Ann. Phys. (N.Y.) 101, 319 (1976); E. Breitenberger, Found. Phys. 15, 353 (1985); J. B. M. Uffink, Phys. Lett. A 108, 59 (1985); J. M. Lévy-Leblond, ibid. 111, 353 (1985); A. Bandilla, H. Paul, and H.-H. Ritze, Quantum Opt. 3, 267 (1991); Z. Hradil, Phys. Rev. A 46, R2217 (1992); Quantum Opt. 4, 93 (1992); T. Opatrný, J. Phys. A 27, 7201 (1994).

[19] U. Larsen, J. Phys. A 23, 1041 (1990); I. Bialynicki-Birula, M. Freyberger, and W. Schleich, Phys. Scr. T48, 113 (1993); M. J. W. Hall, Phys. Rev. A 59, 2602 (1999); Č. Brukner and A. Zeilinger, Phys. Rev. Lett. 83, 3354 (1999); Phys. Rev. A 63, 022113 (2001); J. Řeháček and Z. Hradil, Phys. Rev. Lett. 88, 130401 (2002); A. Luis, Phys. Rev. A 67, 032108 (2003).

[20] A. Rényi, Proceedings of the Fourth Berkeley Symposium on Mathematics and Statistical Probability (University of Califor- nia Press, Berkeley, 1961), Vol. 1, pp. 547-561.

[21] H. Maassen and J. B. M. Uffink, Phys. Rev. Lett. 60, 1103 (1988); E. J. Heller, Phys. Rev. A 35, 1360 (1987); B. Mirbach and H. J. Korsch, Ann. Phys. (N.Y.) 265, 80 (1998); A. Anderson and J. J. Halliwell, Phys. Rev. D 48, 2753 (1993); S. Gnutzmann and K. Życzkowski, J. Phys. A 34, 10123 (2001).

[22] M. O. Scully and M. S. Zubairy, Quantum Optics (Cambridge University Press, Cambridge, England, 1997).

[23] H. Paul, Fortschr. Phys. 22, 657 (1974); J. H. Shapiro and S. S. Wagner, IEEE J. Quantum Electron. 20, 803 (1984); Y. Lai and H. A. Haus, Quantum Opt. 1, 99 (1989); U. Leonhardt and H. Paul, Phys. Rev. A 47, R2460 (1993); J. Mod. Opt. 40, 1745 (1993); M. Freyberger and W. P. Schleich, Phys. Rev. A 47, R30 (1993); M. Freyberger, K. Vogel, and W. P. Schleich, Quantum Opt. 5, 65 (1993); Phys. Lett. A 176, 41 (1993). 